(NASA-CR-199998) MAGNETOSPHERIC FILTER EFFECT FOK PC 3 ALFVEN MODE wAVES (NASA. Marshall Space Flight (enter) $6 \mathrm{p}$
N96-19526

Unclas

$63 / 46 \quad 0101213$ 


\title{
Magnetospheric filter effect for Pc 3 Alfvén mode waves
}

\author{
X. Zhang, ${ }^{1}$ R. H. Comfort, ${ }^{1}$ D. L. Gallagher, ${ }^{2}$ J. L. Green, ${ }^{3}$ Z. E. Musielak, ${ }^{1}$ and \\ T. E. Moore 2
}

Abstract. We present a ray-tracing study of the propagation of Pc 3 Alfvén mode waves originating at the dayside magnetopause. This study reveals interesting features of a magnetospheric filter effect for these waves. Pc 3 Alfven mode waves cannot penetrate to low Earth altitudes unless the wave frequency is below approximately $30 \mathrm{mHz}$. Configurations of the dispersion curves and the refractive index show that the gyroresonance and pseudo-cutoff introduced by the heavy ion $\mathrm{O}^{+}$block the waves. When the $\mathrm{O}^{+}$concentration is removed from the plasma composition, the barriers caused by the $\mathrm{O}^{+}$no longer exist, and waves with much higher frequencies than $30 \mathrm{mHz}$ can penetrate to low altitudes. The result that the $30-\mathrm{mHz}$ or lower frequency Alfvén waves can be guided to low altitudes agrees with ground-based power spectrum observations at high latitudes.

\section{Introduction}

Studies have shown the magnetopause to be an important wave source region for Alfvén mode Pc 3 waves. Guided propagation of Alfven waves in the magnetosphere has generally been discussed in terms of so-called toroidal and transverse poloidal modes of oscillation [Dungey, 1954, 1967]. It has been established that many of the disturbances in the Earth's magnetosphere are a response to variations in the solar wind. Much evidence has shown that plasmas can penetrate through the Earth's bow shock to ionospheric altitudes in the cusp region [Heikkila and Winningham, 1971; Frank, 1982]. There are several possible mechanisms for the generation of Alfven mode waves. One is the perturbation of fluid elements at the magnetopause by a sudden change in solar wind pressure. This change will move the boundary from its equilibrium position, and the disturbance would propagate in the form of Alfvén waves [Sibeck, 1990]. Another wave generation mechanism is the surface wave model, involving the Kelvin-Helmholtz instability at the magnetopause [Miura, 1984, Yumoto et al., 1984]. A third wave generation mechanism is the penetration by upstream ULF waves of the collisionless bow shock region, across the magnetosheath and the magnetopause. Studies have shown that Pc 3 pulsations are related to activity in the ion foreshock region of the solar wind upstream of the Earth's bow shock and magnetosphere [Odera, 1986; Arnoldy et al., 1988; Engebretson et al., 1990].

In a previous paper [Zhang et al., 1993] we examined propagation properties of compressional Pc 3 waves generated in the upstream dayside magnetopause using a ray-tracing analy-

\footnotetext{
${ }^{1}$ Center for Space Plasma and Aeronomic Research, University of Alabama, Huntsville.

${ }^{2}$ Space Sciences Laboratory, NASA Marshall Space Flight Center, Huntsville, Alabama.

${ }^{3}$ National Space Science Data Center, NASA Goddard Space Flight Center, Greenbelt, Maryland.
}

Copyright 1995 by the American Geophysical Union.

Paper number 95JA00276.

0148-0227/95/95JA-00276\$05.00 sis. The results show that the magnetosphere filters the highfrequency components of $\mathrm{Pc} 3$ compressional waves, allowing the low-frequency component waves to penetrate to low altitudes. The penetration of Pc 3 compressional waves is, to a great extent, controlled by the $\mathrm{He}^{+}-\mathrm{O}^{+}$relative concentration. In this paper we present preliminary results of a ray-tracing study of Pc 3 Alfvén mode waves $(20 \mathrm{mHz} \leq f \leq 100 \mathrm{mHz}$ ) started from the dayside magnetopause. We demonstrate that a similar magnetospheric filter effect also applies to Pc 3 Alfven mode waves. We then compare the ray-tracing results with ground observations from the South Pole Station and McMurdo, Antarctica (at invariant latitudes of $-74^{\circ}$ and $-79^{\circ}$, respectively), reported by Engebretson et al. [1989, 1990].

\section{Models for This Study}

In a previous paper [Zhang et al., 1993] we introduced our ray-tracing program and the plasma and magnetic field models. Briefly, the basic ray-tracing equations are from Haselgrove [1954], and this ray-tracing program is based on the Stix [1962] cold plasma index of refraction. In this study we use a modified ray-tracing code from Green et al. [1977]. The original ray-tracing code was written by Shawhan [1967]. The magnetic field model used in this study is from the empirical model of Mead and Fairfield [1975]. The distribution of plasmaspheric plasma density is from the empirical density model of D. L. Gallagher et al. (Composite empirical model of the inner magnetosphere, submitted to Journal of Geophysical Research, 1995), and plasma composition is based on the observations of thermal ion distributions by Horwitz et al. [1986] and Comfort et al. [1988]. For this study we use 75\% $\mathrm{H}^{+}, 20 \% \mathrm{He}^{+}, 5 \% \mathrm{O}^{+}$throughout the magnetosphere.

\section{Characteristics of Dispersion Curves and the Refractive Index for Pc 3 Alfvén Mode Waves}

Figure 1 shows cold plasma dispersion relation curves for the model magnetopause region in the $\mathrm{Pc} 3$ wave frequency range. In the dispersion curves we mark two types of wave modes, Alfvén mode and fast mode. Figure 2 shows contours of the refractive index for the transverse Alfven mode wave in the 


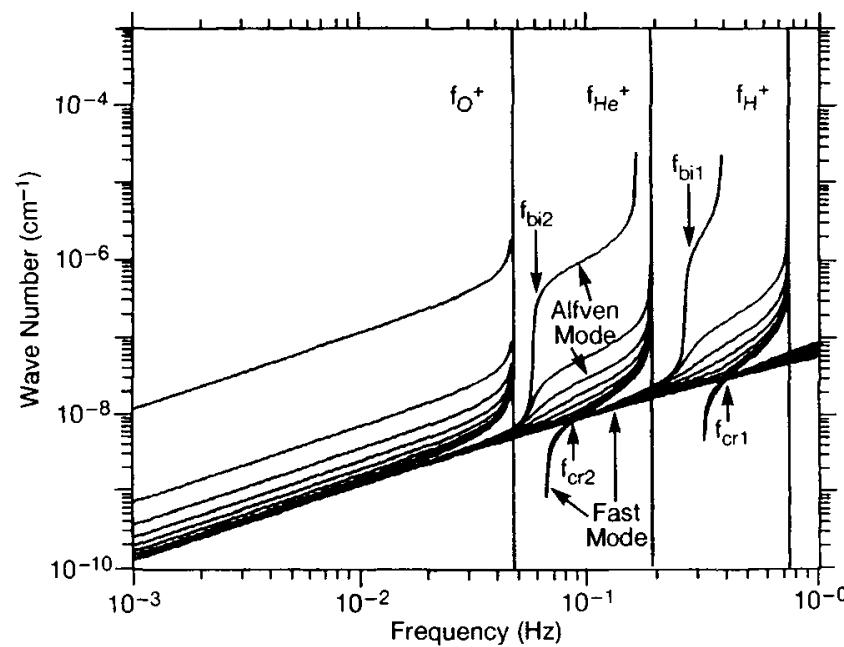

Figure 1. Cold plasma wave dispersion curves for conditions at the equatorial dayside magnetopause (11 $R_{E}$ geocentric).

meridian plane for a frequency of $100 \mathrm{mHz}$. Comparing this topology of the refractive index of Pc 3 Alfven mode waves with the dispersion relation (Figure 1), we can identify three resonance regions in Figure 2 which correspond to the three ion cyclotron resonances for $\mathrm{H}^{+}, \mathrm{He}^{+}$, and $\mathrm{O}^{+}$for $\mathrm{Pc} 3$ Alfvén mode waves. For a fixed frequency, heavier ions will have a cyclotron resonance closer to the Earth, at higher magnetic field strengths, than lighter ions. The $\mathrm{H}^{+}$resonance therefore is found at the largest radial distance from the Earth (in the polar region), the $\mathrm{O}^{+}$resonance is closest to the Earth, and the

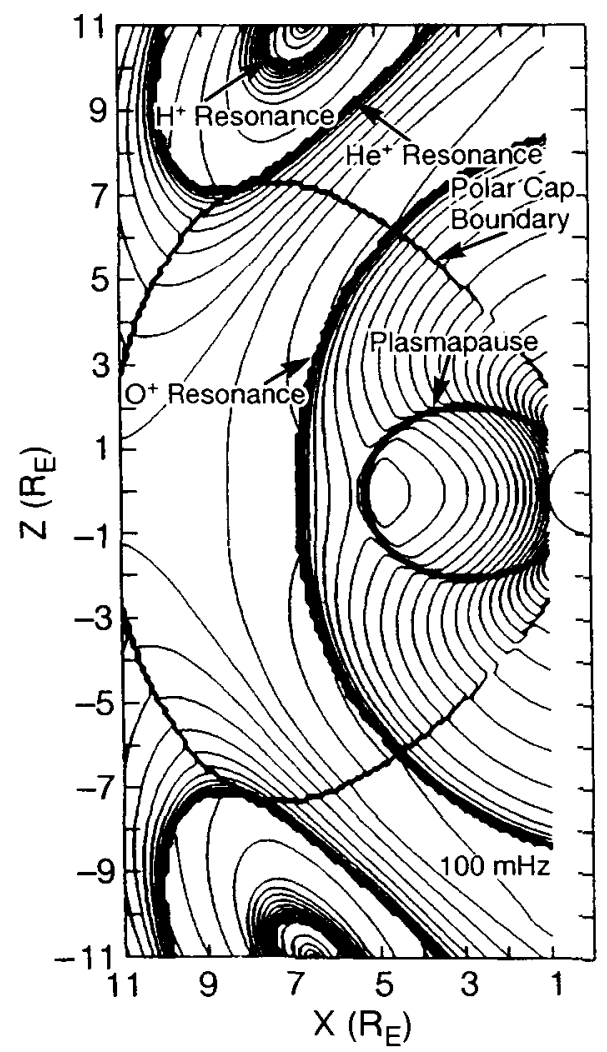

Figure 2. Log scale contours of the refractive index for Alfvén mode waves in the noon-midnight meridian plane $(100$ $\mathrm{mHz}$ ).
$\mathrm{He}^{+}$resonance is in between. The other two intense gradient regions correspond to the plasmapause and to the boundary between the closed field and polar regions. For decreasing wave frequency the resonances will occur at larger radial distances. This is seen in Figure 3 which shows the contours of the refractive index for a wave frequency of $40 \mathrm{mHz}$. Note that the $\mathrm{O}^{+}$resonance no longer encircles the Earth, but is open at low latitudes. The significance of this becomes apparent below.

In order to understand the behavior of Alfven mode waves, we need to discuss some new features besides cutoffs and gyroresonances and their corresponding characteristic frequencies. In the three-ion-species dispersion relation, there are two locations in which the fast mode waves and the Alfven mode waves have the same phase velocity at zero wave normal angle. The corresponding frequency is called a cross-over frequency, defined by Smith and Brice [1964]. For three-ionspecies plasmas we have two cross-over frequencies, which we denote by $F_{\mathrm{cr} 1}$ and $F_{\mathrm{cr} 2}$. These cross-over frequencies occur between the $\mathrm{H}^{+}-\mathrm{He}^{+}$and $\mathrm{He}^{+}-\mathrm{O}^{+}$resonances, respectively. There are two other special locations in the dispersion curves (Figure 1). The first of them is located in the frequency range between the $\mathrm{He}^{+}-\mathrm{O}^{+}$cutoff frequency and $\mathrm{F}_{\mathrm{O}^{+}}$, the $\mathrm{O}^{+}$cyclotron frequency, and the second between the $\mathrm{H}^{+}-\mathrm{He}^{+}$cutoff frequency and $F_{\mathrm{He}^{+}}$, the $\mathrm{He}^{+}$cyclotron frequency. At these two special locations the dispersion curves show steep slopes, similar to resonances and cutoffs. We will refer to them as pseudo-cutoffs (or pseudo-resonances), since their properties are similar to cutoffs or resonances for waves. Their corresponding frequencies are called bi-ion-hybrid frequencies [Rauch and Roux, 1982], which we refer to as $F_{\text {bi1 }}$ and $F_{\text {bi2 }}$, respectively.

We note in Figure 3 (and by inference from Zhang et al.,

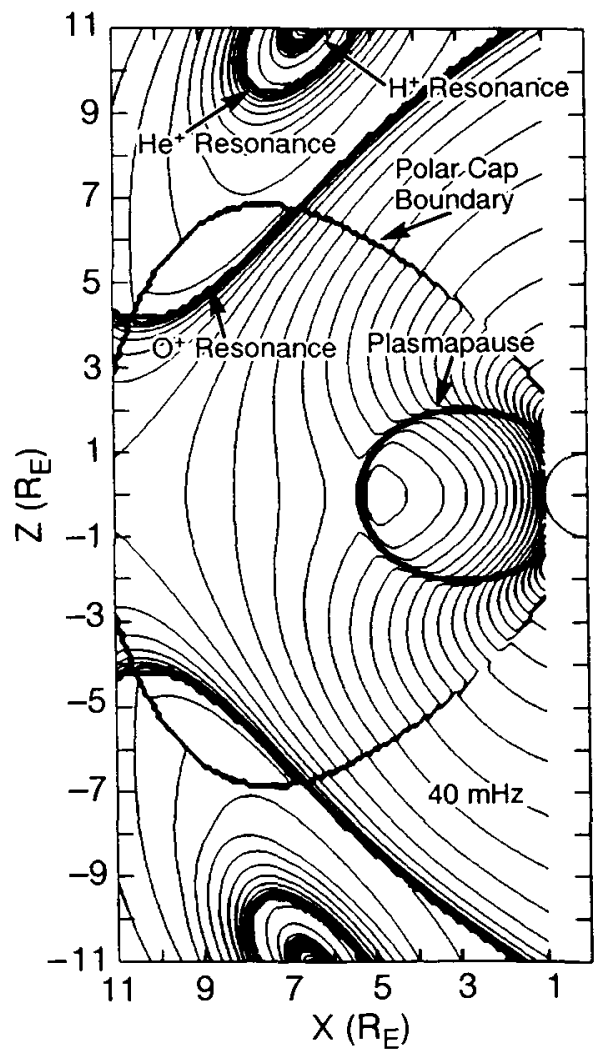

Figure 3. Log scale contours of the refractive index for Alfven mode waves in the noon-midnight meridian plane (40 $\mathrm{mHz}$. 
[1993]) that when the wave frequency is lower than about 40 $\mathrm{mHz}$, the $\mathrm{O}^{+}$resonance band becomes open at low latitudes. This frequency $(40 \mathrm{mHz})$ is near the "critical frequency" for Pc 3 Alfvén mode waves. Similar to that defined by Zhang et al. [1993], this critical frequency is defined as the frequency for which the $\mathrm{O}^{+}$resonance location just becomes tangent to the magnetopause at the equator. The value of this frequency depends on the magnitude of the geomagnetic field at the magnetopause. As such, the critical frequency will vary with solar wind conditions and may vary with the model of the geomagnetic field being employed. As the field intensity increases the critical frequency will also increase.

Waves with frequencies below the critical frequency are located leftward of $\mathrm{F}_{\mathrm{O}^{+}}$in the dispersion relation curves (Figure 1). Since the general tendency for these waves is to travel toward the intense magnetic field strength region along field lines, the dispersion curves move to the right relative to the wave frequency. Therefore Pc 3 Alfven mode waves below the critical frequency (or below the local $\mathrm{O}^{+}$gyrofrequency) are free from all cutoff or resonance (including pseudo-cutoff or pseudo-resonance) barriers. These waves are able to penetrate to the Earth's ionosphere. Unlike the situation of cutoffs for fast mode waves, the locations of the gyroresonances do not vary with the plasma relative concentrations. However, if we eliminate an ion species, say $\mathrm{O}^{+}$, the corresponding resonance disappears. The influence of pseudo-resonances is discussed further below.

\section{Ray-Tracing Examples of Alfvén Mode Waves}

In the Figures below, we present families of ray paths for Pc 3 transverse Alfvén mode waves. All rays are launched from

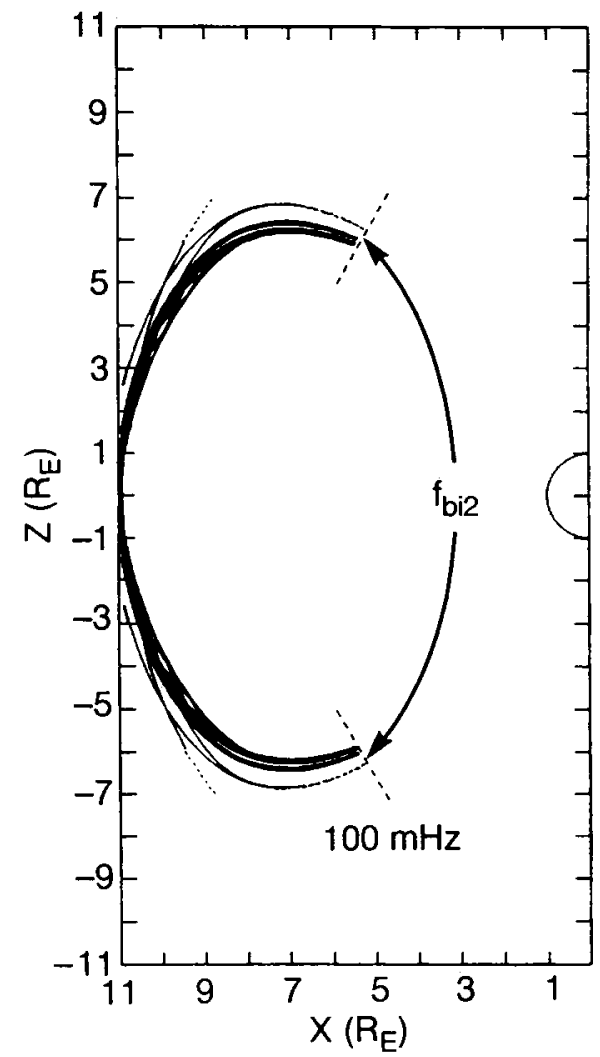

Figure 4. Meridian ray paths for $100-\mathrm{mHz}$ Alfvén mode waves starting at the equatorial dayside magnetopause (11 $R_{E}$ geocentric).

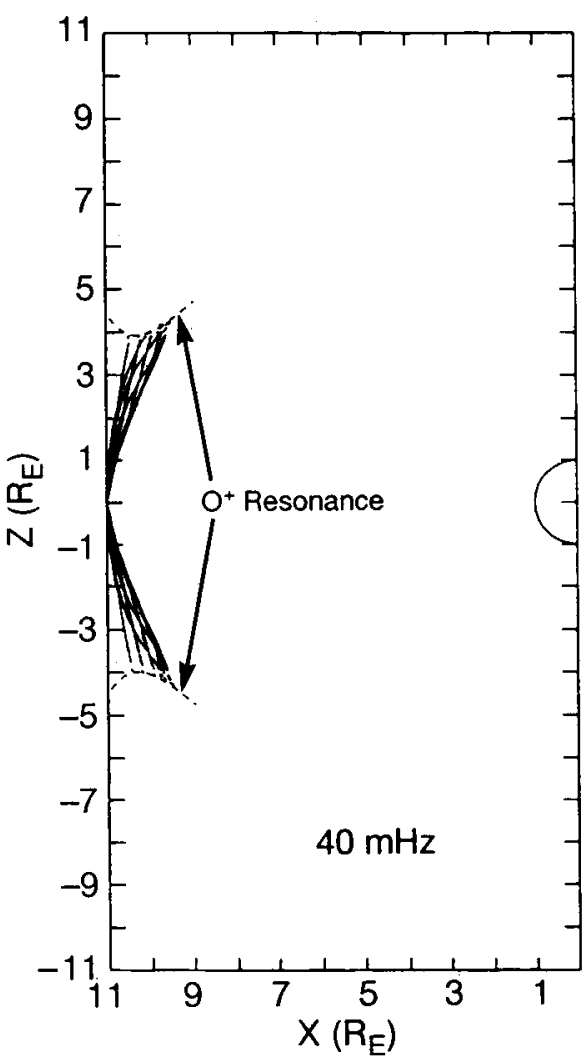

Figure 5. Meridian ray paths for $40-\mathrm{mHz}$ Alfven mode waves starting at the equatorial dayside magnetopause (11 $R_{E}$ geocentric).

the magnetic equator on the dayside magnetopause. Rays are launched in the noon-midnight magnetic meridian plane at varying initial wave normal angles. We use a solid line for rays with WKB condition [Zhang et al., 1993] equal to or less than 0.1 and a dashed line when the WKB condition is greater than 0.1 . A solid line means that the ray tracing well satisfies the WKB approximation; a dashed line gives a warning for the WKB approximation.

Figures 4, 5, and 6 show rays of transverse Alfvén waves with frequencies of $100 \mathrm{mHz}, 40 \mathrm{mHz}$, and $30 \mathrm{mHz}$. We display rays launched with a range of initial wave normal angles from $0^{\circ}$ to $180^{\circ}$, at $10^{\circ}$ intervals. We can see that Pc 3 Alfvén mode waves are guided by the magnetic field line, as is characteristic of Alfven waves and required by the index of refraction. From Figure 4 the $100-\mathrm{mHz}$ rays are guided along magnetic field lines to the increased magnetic field strength region so that each of the gyrofrequencies increases. In the dispersion relation (Figure 1) we can see that the $100-\mathrm{mHz}$ wave frequency is between $F_{\mathrm{O}^{+}}$and $F_{\mathrm{He}^{+}}$. As the magnetic field increases along the wave trajectory, the dispersion curves in Figure 1 move to the right. At the location where $F=F_{\mathrm{cr} 2}$, the waves undergo a polarization reversal and are converted from left-hand polarized to right-hand polarized waves. As the waves move farther along the dispersion curve, they encounter the pseudocutoff at the point $F=F_{\text {bi2 }}$, the second bi-ion-hybrid frequency, and waves are reflected. If we compare this ray plot with the contours of the refractive index for both fast mode [Zhang et al., 1993] and Alfvén mode (Figure 2) waves for this same frequency, we find that the location of reflection for 100 $\mathrm{mHz}$ in Figure 4 is between the $\mathrm{He}^{+}-\mathrm{O}^{+}$cutoff and the $\mathrm{O}^{+}$ gyroresonance. This position just corresponds to the location 


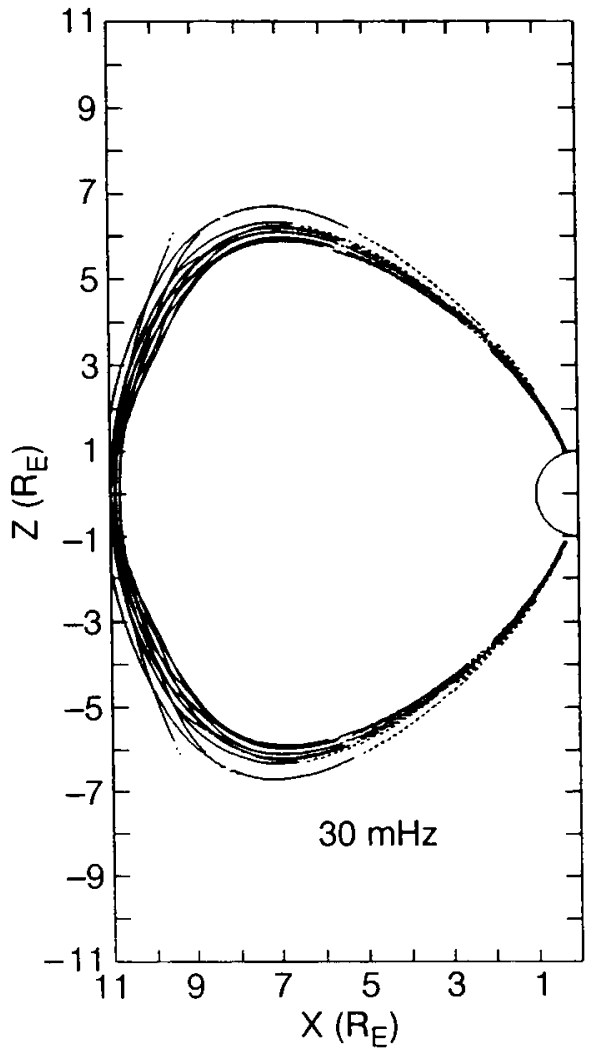

Figure 6. Meridian ray paths for $30-\mathrm{mHz}$ Alfvén mode waves starting at the equatorial dayside magnetopause (11 $R_{E}$ geocentric).

of the pseudo-cutoff in the dispersion relation (Figure 1). In this case the range of frequencies at which waves are reflected back to the magnetopause depends on the relative concentrations of $\mathrm{He}^{+}$and $\mathrm{O}^{+}$. Reflection will occur at higher frequencies as a result of an increasing ratio of $\mathrm{O}^{+}$to $\mathrm{He}^{+}$concentrations.

The situation for $40-\mathrm{mHz}$ rays in Figure 5 is quite different. This frequency $(40 \mathrm{mHz})$ is just slightly below $F_{\mathrm{O}^{+}}$in the dispersion relation (Figure 1). These Alfvén mode waves propagate along an $L$ shell into a region of decreasing magnetic field strength (a feature of the realistic dayside magnetic field). In the dispersion relation (Figure 1) the gyrofrequencies move leftward, showing that the wave will propagate into the $\mathrm{O}^{+}$ gyroresonance and be absorbed. If we overlay the contours of the refractive index for $\mathbf{4 0} \mathrm{mHz}$ for Alfvén mode waves (Figure 2) with the ray trajectory traces (Figure 5), we can see that these rays stop at the location where they encounter the $\mathrm{O}^{+}$ gyroresonance.

The $30-\mathrm{mHz}$ rays in Figure 6 are below the critical frequency. The wave source is in a location below $F_{\mathrm{O}^{+}}$in the dispersion relation (Figure 1). When these waves propagate along magnetic field lines, they narrowly miss (individual rays encounter) the $\mathrm{O}^{+}$gyroresonance. After these rays pass the region of decreasing magnetic field strength they enter a region of increasing magnetic field strength, so that the wave locations move leftward along dispersion curves in Figure 1 (in the dispersion relation (Figure 1) the gyrofrequencies move rightward). Therefore whe. the rays escape from the $\mathrm{O}^{+}$ gyroresonance, they are also free from all the cutoff or resonance (including pseudo-cutoff or pseudo-resonance) barriers at frequencies above $F_{0^{+}}$. Therefore most $30-\mathrm{mHz}$ rays are able to be guided along magnetic field lines to the Earth's ionosphere.
Since an $\mathrm{O}^{+}$concentration in the magnetosphere introduces cutoff and resonance (including pseudo-cutoff or pseudoresonance) barriers in the magnetosphere in the Pc 3 frequency range, it is of interest to check the case where there is no $\mathrm{O}^{+}$ concentration. Figure 7 shows Alfvén mode rays at $100 \mathrm{mHz}$ with no $\mathrm{O}^{+}$, where the concentration of $\mathrm{H}^{+}$is $80 \%$ and $\mathrm{He}^{+}$is $20 \%$. Because the $\mathrm{O}^{+}$concentration is removed, all the cutoffs and resonances (including pseudo-cutoffs or pseudoresonances) at frequencies below $F_{\mathrm{He}^{+}}$in the dispersion relation (Figure 1) are gone. There are no more barriers for 100 $\mathrm{mHz}$ Alfven mode waves, so they are able to penetrate to the Earth's ionosphere. This plot further supports our suggestion that $\mathrm{O}^{+}$is the main factor which keeps Pc 3 transverse Alfvén waves from reaching the ionosphere.

\section{Discussion and Summary}

There have been extensive experiments and theoretical reports [e.g., Anderson et al., 1990] on the role which Pc 3 waves $(20-100 \mathrm{mHz})$ play in energy transport from the solar wind to the inner magnetosphere. However, the fundamental question as to how wave energy is transported from the solar wind in the foreshock magnetosheath to the low-altitude magnetosphere remains unclear. Our previous ray-tracing study [Zhang et al, , 1993] revealed important features regarding transport of wave energy from the magnetopause into the inner magnetosphere through Pc 3 compressional waves. This study reveals magnetospheric wave energy transport through the other wave mode branch, Pc 3 Alfven mode waves.

The penetration of upstream ULF magnetic pulsations across the magnetopause was studied by Troitskaya et al. [1971], Hoppe and Russell [1983], and Yumoto et al. [1984].

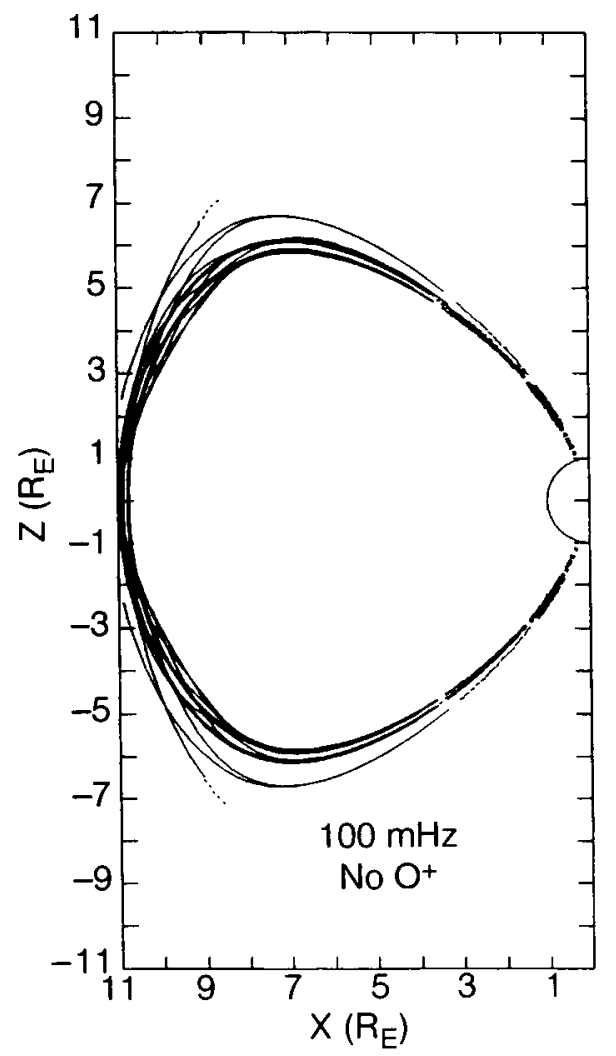

Figure 7. Meridian ray paths for $100-\mathrm{mHz}$ Alfvén mode waves (in a magnetosphere without $\mathrm{O}^{+}$) starting at the equatorial dayside magnetopause (11 $R_{E}$ geocentric). 
Yumoto et al. [1984] suggested that the magnetopause wave source of high-latitude Pc 3 pulsations can be either surface waves excited by solar wind-driven instabilities or upstream waves in the Earth's foreshock. Observations support the idea that wave energy could be transported via cusp field lines from the magnetopause to the ionosphere through Pc 3 Alfvén mode wave propagation [Bol'shakova and Troitskaya, 1984; Plyasova-Bakounina et al., 1986; Morris and Cole, 1987; Engebretson et al., 1989]. Our ray-tracing study of Pc 3 Alfvén mode waves provides a more concrete picture of how wave energy at the magnetopause might be transported to the highlatitude ionosphere and also what constraints on this transport might be imposed by the magnetospheric plasma medium.

This study shows that the dominant behavior of the Earth's magnetosphere is like that of a low pass filter for transverse Alfvén waves generated at the equatorial magnetopause. Figure 8 shows schematically the characteristics of the filter action of the magnetosphere for Pc 3 transverse Alfvén mode waves. The vertical coordinate is wave frequency, and the horizontal coordinate is the penetration altitude. This Figure shows that when the wave frequency is below about $30 \mathrm{mHz}$, Pc 3 Alfvên mode waves are able to penetrate to the $1-R_{E}$ (ionosphere) region, so the passband to the ionosphere for $\operatorname{Pc} 3$ transverse Alfven mode waves is below $30 \mathrm{mHz}$. There is also a stop band in the characteristics of the filter. The stop band is from about $40 \mathrm{mHz}$ to about $60 \mathrm{mHz}$, for which there are no Alfvén mode waves. The reason for the existence of the stop band is that between the gyrofrequency and bi-ion cutoff, Alfven mode waves do not exist. At somewhat higher frequencies Alfvén mode waves can propagate into the magnetosphere before being turned back by the $\mathrm{He}^{+}-\mathrm{O}^{+}$ion-ion resonance. Just above this band and above the lower frequency transmission band, the Alfven mode wave encounters either the $\mathrm{He}^{+}$or the $\mathrm{O}^{+}$gyroresonance and is absorbed. Of course, the frequency spectrum which is actually observed at a given location depends on the source spectrum as well as the propagation characteristics.

The results from our ray-tracing study match very well with high-latitude ground station observations. Engebretson et al. $[1989,1990]$ reported that $P c 3$ wave activity was observed at South Pole Station, which is located at a latitude near the

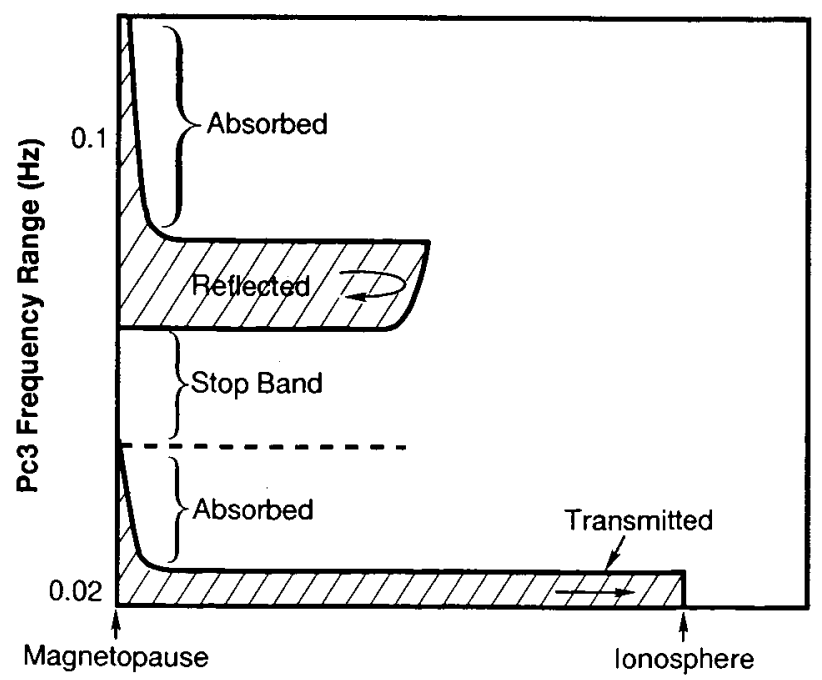

Radial Distance

Figure 8. Schematic characteristics of the filter action of the magnetosphere for Pc 3 Alfvén mode waves. nominal footpoint of the dayside cusp/cleft region, and McMurdo, Antarctica (at invariant latitudes of $-74^{\circ}$ and $-79^{\circ}$, respectively). During selected days in March and April 1986, Pc 3 activity correlated with the low interplanetary magnetic field (IMF) cone angle, suggesting that this Pc 3 activity was related to upstream wave activity. The power spectra observed at these two high-latitude observatories have a steep drop-off in spectral density toward high frequencies. The cutoff frequency in the passband of the power spectrum is near $30 \mathrm{mHz}$, which is consistent with the results from our ray-tracing studies. As noted above, the observed drop-off in wave frequency could be associated with the source mechanism for the waves. To determine whether source characteristics could also be responsible would require comparison of wave spectral observations at both high and low altitudes along the appropriate field lines. However, it would be an interesting coincidence if both source and propagation mechanisms gave similar observational consequences at low altitudes.

From the above analysis we can conclude that the cold plasma approximation is sufficient to explain a low-pass effect for Alfven mode waves propagating from the equatorial magnetopause to the ionosphere. The passband is located below about $30 \mathrm{mHz}$; this frequency is a function of the magnetopause magnetic field strength. That means that increased solar wind pressure should raise the magnetic field strength there and increase the maximum frequency of Pc 3 waves observed on the ground. The characteristics of the magnetospheric filter are largely determined by the magnetic field strength at the magnetopause and along the magnetopause $L$ shell. However, removal of the $\mathrm{O}^{+}$concentration in our plasma model dramatically enlarges the passband of the filter. In that case the magnetosphere lets almost the full frequency range of Pc 3 transverse Alfven waves pass to the ionosphere.

We anticipate that Alfvén mode waves will also be able to reach the ionosphere for very low, nonzero concentrations of $\mathrm{O}^{+}$. A measure of the likelihood of tunneling between the $\mathrm{He}^{+}-$ $\mathrm{O}^{+}$bi-ion cutoff frequency and the $\mathrm{O}^{+}$gyrofrequency is taken to be when the required tunneling spatial distance is equal to the wavelength of the Alfven waves. For the situation shown in Figure $4,100-\mathrm{mHz}$ waves are reflected away from the Earth at a distance of about $8 R_{E}$. At the bi-ion cutoff, Alfven waves have a wavelength of about $0.1 R_{E}$. We find the tunneling distance will be the same as the wavelength when the ratio of the $\mathrm{O}^{+}$to $\mathrm{He}^{+}$relative concentrations falls below 0.04 . If $\mathrm{H}^{+}$has a concentration of $75 \%$, as used in this work, then tunneling is expected to become important for concentrations of $\mathrm{O}^{+}$less than about $1 \%$ of the total density.

Since the cleft ion fountain is known to pump heavy ions into the magnetosphere, the question arises as to whether heating and associated upwelling $\mathrm{O}^{+}$could effectively change the Pc 3 wave characteristics observed in the ionosphere. The answer is probably not. First, to make a change, the dayside cleft flux tubes would have to initially contain no $\mathrm{O}^{+}$at high altitudes, and then suddenly have $\mathrm{O}^{+}$flow up to sufficiently high altitudes (low magnetic field intensity) that its gyrofrequency could resonate with the wave. Since ions flowing up the cleft flux tubes are typically convecting plasma toward the nightside, and the heavier ions are convected more strongly than the lighter ions due to the mass spectrometer dispersive effect, it is not likely that $\mathrm{O}^{+}$would reach the necessary altitudes on the dayside to have a significant effect, from this type of mechanism.

Of course this mechanism is not limited to the dayside. At 
any location where the waves are propagating, where $\mathrm{O}^{+}$is present, and where the magnetic field magnitude is at the right level, significant effects on the propagation could be produced by this filter mechanism. If it is operating, the observable effect would be a broader spectrum of waves (at the high end) at magnetospheric altitudes than at ionospheric altitudes.

The characteristics of the Pc 3 Alfven mode waves are quite analogous to those of Pc 3 compressional fast mode waves. Our previous study [Zhang et al., 1993] also reveals the lowpass feature of the magnetosphere to the Pc 3 compressional fast mode waves. The mechanism of this low-pass feature for Pc 3 compressional fast mode waves is also attributed to the existence of the $\mathrm{O}^{+}$concentration in the magnetosphere, which along with the $\mathrm{He}^{+}$concentration produces the $\mathrm{He}^{+}-\mathrm{O}^{+}$ cutoff barrier to the propagation of the Pc 3 compressional fast mode waves. The difference between the propagation characteristics of Pc 3 Alfvén mode waves and Pc 3 compressional fast mode waves is that the Alfvén mode waves could propagate to the high-latitude ionosphere along the magnetic field line, while the compressional fast mode waves could penetrate to the low-latitude plasmasphere, if their frequencies were all lower than corresponding critical frequencies. Another difference is that the propagation characteristics of Pc 3 Alfvén mode waves are less sensitive to the relative concentrations of helium and oxygen ions than are the Pc 3 compressional fast mode waves.

Acknowledgments. This work of X. Zhang and R. H. Comfort was partially supported by NASA grants NAGW-1630 and NAG8-239, and Z. E. Musielak was partially supported by NSF under grant ATM9119580. Work at MSFC was supported in part by NASA Space Physics Division RTOP 432-20-00.

The Editor thanks J. L. Rauch and R. D. Elphinstone for their assistance in evaluating this paper.

\section{References}

Anderson, B. J., M. J. Engebretson, S. P. Rounds, L. J. Zanetti, and T. A. Potemra, A statistical study of Pc 3-5 pulsations observed by the AMPTE/CCE magnetic fields experiment 1, Occurrence distributions, J. Geophys. Res., 95, 10,495, 1990.

Amoldy, R. L., L. J. Cahill Jr., M. J. Engebretson, L. J. Lanzerotti, and A. Wolfe, Review of hydromagnetic wave studies in the Antarctic, Rev. Geophys., 26, 181, 1988.

Bol'shakova, O. V., and V. A. Troitskaya, The relation of the highlatitude maximum of Pc 3 intensity to the dayside cusp, Geomagn. Aeron. Engl. Transl., 24, 633, 1984.

Comfort, R. H., I. T. Newberry, and C. R. Chappell, Preliminary statistical survey of plasmaspheric ion properties from observations by $D E$ 1/RIMS, in Modeling Magnetospheric Plasma, Geophys. Monogr. Ser., vol. 44, edited by T. E. Moore and J. H. Waite Jr., pp. 107-114, AGU, Washington, D.C., 1988.

Dungey, J. W., Electrodynamics of the Outer Atmosphere, Ionosphere Res. Lab., Pennsylvania State Univ., University Park, PA, 1954.

Dungey, J. W., Hydromagnetic waves, in Physics of Geomagnetic Phenomena, edited by S. Matsushita and W. H. Campbell, 913 pp., Academic, New York, 1967.

Engebretson, M. J., B. J. Anderson, L. J. Cahill Jr., R. L. Amoldy, P. T. Newell, C.-I. Meng, L. J. Zanetti, and T. A. Potemra, A multipoint case study of high-latitude daytime ULF pulsations, J. Geophys. Res., 94, 17,143, 1989.

Engebretson, M. J., B. J. Anderson, L. J. Cahill, Jr., R. L. Arnoldy, T. J. Rosenberg, D. L. Carpenter, W. B. Gail, and R. H. Eather, Ionospheric signatures of cusp latitude Pc 3 pulsations, J. Geophys. Res., 95, 2447, 1990.
Frank, L. A., Plasma in the Earth's polar magnetosphere, J. Geophys. Res., 87, 4560, 1982.

Green, J. L., D. A. Gurnett, and S. D. Shawhan, The angular distribution of auroral kilometric radiation, J. Geophys, Res., 82, 1825, 1977.

Haselgrove, J., Ray Theory and a new method for ray tracing, in Report of Conference on the Physics of the Ionosphere, 355 pp., London Phys. Soc., 1954.

Heikkila, W. J., and J. D. Winningham, Penetration of magnetosheath plasma to low altitudes through the dayside magnetospheric cusps, $J$. Geophys. Res., 76, 883, 1971.

Hoppe, M. M., and C. T. Russell, Plasma rest frame frequencies and polarizations of the low-frequency upstream waves: ISEE 1 and 2 observations, J. Geophys. Res., 88, 2021, 1983.

Horwitz, J. L., R. H. Comfort, and C. R. Chappell, Plasmasphere and plasmapause characteristics measured by DE-1, Adv. Space Res., $6(3), 21,1986$.

Mead, G. D., and D. H. Fairfield, A quantitative magnetospheric model derived from spacecraft magnetometer data, J. Geophys., Res., 80, $523,1975$.

Miura, A., Anomalous transport by magnetohydrodynamic KelvinHelmholtz instabilities in the solar wind-magnetosphere interaction, J. Geophys. Res., 89, 801, 1984.

Morris, R. J., and K. D. Cole, Pc 3 magnetic pulsations at Davis, Antarctica, Planet. Space Sci., 35, 1437, 1987.

Odera, T. J., Solar wind controlled pulsations, a review, Rev. Geophys., 24, 55, 1986.

Plyasova-Bakounina, T. A., V. A. Troitskaya, J. W. Muench, and H. F. Gauler, Super-high-latitude maximum of Pc 2-4 intensity, Acta Geod. Geophys. Montan. Hung., 21, 1986.

Rauch, J. L., and A. Roux, Ray tracing of ULF waves in a multicomponent plasma: Consequence for the generation mechanism of ion cyclotron waves, J. Geophys. Res., 87, 8191, 1982.

Shawhan, S. D., Behavior of VLF Ray Paths in the Ionosphere, University of Iowa Press, Iowa City, IA, 1967.

Sibeck, D. G., A model for transient magnetospheric response to sudden solar wind dynamic pressure variations, J. Geophys. Res., 95, 3755 , 1990.

Smith, R. L., and N. Brice, Propagation in multicomponent plasmas, $J$. Geophys. Res., 69, 5029, 1964.

Stix, T. H., The Theory of Plasma Waves, McGraw-Hill, New York, 1962.

Troitskaya, V. A., T. A. Plyasova-Bakounina, and A. V. Gul'elmi, Relationship between $\mathrm{Pc} 2-4$ pulsations and the interplanetary magnetic field, Dokl. Akad. Nauk. SSSR, 197, 1312, 1971.

Yomoto, K., A. Wolfe, T. Terasawa, E. L. Kamen, and L. J. Lanzerotti, Dependence of $\mathrm{Pc} 3$ magnetic energy spectra at south pole in upstream solar wind parameters, Planet Space Sci., 32, 1205, 1984.

Zhang, X., R. H. Comfort, Z. E. Musielak, T. E. Moore, D. L. Gallagher, and J. L. Green, Propagation characteristics of Pc 3 compressional waves generated at dayside magnetopause, J. Geophys. Res., 98 , 15,403, 1993.

R. H. Comfort, Z. E. Musielak, and X. Zhang, Center for Space Plasma and Aeronomic Research, University of Alabama in Huntsville, Huntsville, AL 35899. (e-mail: comfort @espar.uah.edu)

D. L. Gallagher and T. E. Moore, Space Sciences Laboratory, Mail Code ES83, NASA Marshall Space Flight Center, Huntsville, AL 35812. (e-mail:dennis.gallagher@msfc.nasa.gov; thomas.moore@msfc.nasa. gov)

J. L. Green, National Space Science Data Center, Mail Code 630, NASA Goddard Space Flight Center, Greenbelt, MD 20771. (email:green @nssdca.gsfc.nasa.gov)

(Received September 15, 1994; revised January 20, 1995; accepted January 23, 1995.) 